\title{
2 Pedaling time variability is increased in dropped riding position
}

\author{
Johnny Padulo • Roberto Di Capua • \\ Davide Viggiano
}

5 Received: 15 September 2011/ Accepted: 9 December 2011

6 (c) Springer-Verlag 2011

7 Abstract Variability of cycle-to-cycle duration during a 8 pedaling task is probably related to the rhythmic control of 9 the lower limb muscles as in gait. Although walking var10 iability has been extensively studied for its clinical and 11 physiological implications, pedaling variability has 12 received little attention. The present contribution deter13 mines the variability of the cycling time during a 10-min 14 exercise as a function of upper body position. Nine healthy 15 males were required to pedal on cycle-ergometer at a self16 selected speed for $10 \mathrm{~min}$ in two different upper body 17 positions [hands on upper handlebars (UP) or lower han18 dlebars (DP)]. Time domain measures of cycling variability 19 [total standard deviation (SDtot), mean standard deviation 20 cycle-to-cycle intervals over
100 cycles (SD100), standard deviation of the average cycle-to-cycle intervals over 100 cycles (SDA100)] were measured. Moreover, the same time domain measures were also calculated for heart rate in order to discriminate possible involvements of autonomic regulation. Finally, the structure of the cycle variations has been analyzed in the framework of deterministic chaos calculating the maximum Lyapunov exponents. Significant increases in cycleto-cycle variability were found for SDtot, SD100 in DP compared to UP, whereas cardiac parameters and other cycling parameters were not changed in the two positions.

Communicated by David C. Poole.

\section{J. Padulo}

Faculty of Medicine and Surgery, Motor Sciences,

University "Tor. Vergata", Rome, Italy

R. Di Capua · D. Viggiano $(\bowtie)$

Department of Health Sciences, University of Molise, Via De Sanctis III Edificio Polifunzionale, 86100 Campobasso, Italy e-mail: davide.viggiano@unimol.it
Moreover, the maximum Lyapunov exponent was significantly more negative in DP. The results suggest that small perturbations of upper body position can influence the control of cycling rhythmicity by increasing the variability in a dissipative deterministic regimen.

Keywords Long-range correlations · Variability · Fatigue · Motor control · Maximum Lyapunov exponent

\section{Introduction}

Cycling is a complex task involving the coordination of lower limbs, and requiring the organization of physiological muscle responses to the environment during races. To this aim, subjects need to adequately explore the immediate environment, and correct the cycling time to appropriate target values. It is taught that, in other movement types such as walking, stride-to-stride variability emerges as a consequence of system's need to continuously correct movement errors (Jordan et al. 2007; Meardon et al. 2011). The study of walking variability has received great attention because it is interesting parameter for pathological conditions such as aging, neuropsychiatric diseases, Parkinson's disease, cruciate ligament deficit (Hausdorff 2009). Therefore, stride time variability during walking and running has been widely studied (Hausdorff et al. 1995a, b; Hausdorff 2009). Unfortunately, pedal cycling variability has received little attention. Cycling at a specific, self-selected, pacing requires the subject to continuously adjust the force produced and its timing relative to the pedal position. When the timing or the module of the force is not applied appropriately, an unwanted acceleration or deceleration of the pedal occurs, inducing a fluctuation in cycle duration. It is possible that unusual riding

\begin{tabular}{|l|lll|}
\hline & Journal $:$ Large $\mathbf{4 2 1}$ & Dispatch : $\mathbf{1 3 - 1 2 - 2 0 1 1}$ & Pages : $\mathbf{5}$ \\
Article No. : $\mathbf{2 2 8 2}$ & $\square \mathrm{LE}$ & $\square$ TYPESET \\
& MS Code : EJAP-D-11-00984 & $\boldsymbol{V}_{\mathrm{CP}}$ & $\checkmark$ DISK \\
\hline
\end{tabular}


positions change cycling variability both due to fatigue/ discomfort or to mechanical factors (Corbeil et al. 2003; Gates and Dingwell 2008; Jordan et al. 2007). Therefore, an increase in the number of corrections of the pedal velocity through timing activation of lower leg muscles is expected to increase cycling variability, possibly as a function of cycling speed.

The present study has been designed to test the hypothesis that, in comparison with standard postures (UP), drop position (DP) would modify the coordination of lower limb muscles during pedaling and consequently would influence the motor control during pedaling, thus changing the pedaling variability.

\section{Methods}

\section{Subjects}

Nine voluntary male subjects (age $41.0 \pm 8.1$ years, height $171 \pm 7.5 \mathrm{~cm}$, weight $66.0 \pm 7.5 \mathrm{~kg}$; mean $\pm \mathrm{SD}$ ) participated to this study. The subjects were healthy without any muscular, neurological and tendineous injuries and did not report any consumption of drugs. After being informed of the procedures, methods, benefits and possible risks involved in the study, each subject reviewed and signed an informed consent to participate in the study. The experimental protocol was performed in accordance with the ethical standards laid down in the Declaration of Helsinki for human experimentation.

\section{Procedures}

Each subject performed a standardized 5-min warm up, consisting of free pedaling on a spinning bike (Schwinn, Johnny G Pro Spin Bike; crank length: $17 \mathrm{~cm}$ ), wearing low-heeled athletic shoes. All subjects were then invited to pedal, in seated position, at a freely chosen cadence. They were required to pedal in two different positions of the upper body: with hands on top of the upper handlebars, near the stem and elbow angle between $160^{\circ}$ and $180^{\circ}$ (UP) or the traditional racing position with the torso partially to fully bent-over, hands on the drops portion of the handlebars and elbows partially flexed (DP; elbow angle less than $160^{\circ}$ ) in according to (Dorel et al. 2009).

Each session lasted 10 min. Between the two sequences subjects could recover for $5 \mathrm{~min}$. The order of the body position was randomized across subjects. To study cycling variability, the crank angular position was measured with a sampling frequency of $100 \mathrm{~Hz}$ using a linear encoder connected to the pedal (MuscleLabTM 4020e, Bosco System, Ergotest Technology, Langensund, Norway; spatial resolution of $0.1 \mathrm{~mm}$ ), which recorded the vertical displacement of the pedal. Moreover, a previous observation showed that cycling modulates the cardiac chronotropic response to exercise, inducing a new component in heart rate variability (Blain et al. 2009). Therefore, we evaluated a possible connection between cycling variability and heart rate variability. To this aim heart function was monitored by measuring heart rate and the duration of each heart beat throughout the experiment, using a PE 3000 Sport Tester (Polar Electro, Kempele, Finland).

\section{Cycling variability analysis}

To analyze the variability of the cycle duration, two approaches have been used: the classical calculation of the variability around the average cycle, and the maximum Lyapunov exponent (LyE) within the framework of the dynamical system theory. The latter has the advantage to further characterize the origin of the variability.

The standard deviation of cycle-to-cycle intervals (SDtot), the average standard deviation cycle-to-cycle intervals over 100 cycles (SD100), the standard deviation of the average cycle-to-cycle intervals over 100 cycles (SDA100) and the average cycle duration were obtained as time domain measures. Similarly, the same time domain measures were also applied for $\mathrm{R}-\mathrm{R}$ interval variability analysis.

The mathematical approach of LyE is based on an infinite amount of data, whereas our time series derives from 10-min observation (about 600 cycles). Moreover, the noise within the dataset also represents a challenge for LyE calculation from limited dataset (for a revision of the application of LyE for human movement see e.g. Sterigou and Decker 2011). Details of the calculation of the LyE can be found in Rosenstein et al. (1992). Briefly, after representation of the data into State Space visualization, False Nearest Neighbors Statistic was used to estimate the number of embedding dimensions. The maximum Lyapunov exponent was then calculated using custom software for each subject in each position.

Statistical analysis

The results are expressed as mean \pm standard error. $t$ student tests for paired data were used to compare the two body positions. The rejection level was set at $p \leq 0.05$.

\section{Results}

All subjects completed the exercise test without any clinical abnormality. However, some subject reported subjective discomfort when pedaling for $10 \mathrm{~min}$ in dropped (DP) posture.

\begin{tabular}{|c|c|c|c|c|}
\hline & Journal : Large 421 & Dispatch & 13-12-2011 & Pages : \\
\hline & $\begin{array}{l}\text { Article No. : } \mathbf{2 2 8 2} \\
\text { MS Code : } \\
\text { EJAP-D-11-00984 }\end{array}$ & $\begin{array}{l}\square \mathrm{LE} \\
\boldsymbol{\iota}_{\mathrm{CP}}^{\mathrm{LE}}\end{array}$ & & $\begin{array}{l}\square \text { TYPESET } \\
\boldsymbol{\vee} \text { DISK }\end{array}$ \\
\hline
\end{tabular}


An exemplificative plot of cycle-to-cycle duration over several pedaling cycles for UP and DP position is shown in Fig. 1a, and the frequency histogram of different cycling durations is shown in the inset: it is evident that in DP posture the frequency histogram shows a larger distribution of pedaling durations. Average cycle duration is reported in Fig. 1b and was not significantly different between the two upper body positions. The analysis of pedaling variability in the two body positions (Fig. 1c-e) showed that the position with the hands on dropped handlebars (DP) increased pedaling variability compared to UP position: the standard deviation of cycle-to- cycle intervals (SDtot) and the average standard deviation cycle-to-cycle intervals over 100 cycles (SD100) were significantly greater in DP position compared to UP position as assessed by two tails $t$ test for paired data ( $p<0.05$; Fig. 1c, d). Conversely, the standard deviation of the average cycle-tocycle intervals over 100 cycles (SDA100) did not significantly change in two positions (Fig. 1e).

The heart rate at the end exercise was not affected by the upper body position during 10-min cycling, as reported in Fig. 2a. Moreover, riding position did not significantly affect heart rate variability (HRV) (Fig. 2).
Fig. 1 Analysis of pedaling variability in two upper body positions during a $10-\mathrm{min}$ cycling exercise.

a Representative plot of cycleto-cycle duration over several pedaling cycles in two riding positions (UP upper handlebars, $D P$ lower handlebars); the inset show the frequency histogram of different pedaling durations. b Average cycle duration. c-e Pedaling variability in two upper body positions; c standard deviation of cycle-to-cycle intervals (SDtot), $\mathbf{d}$ average standard deviation cycle-tocycle intervals over 100 cycles (SD100), e standard deviation of the average cycle-to-cycle intervals over 100 cycles (SDA100). $\mathbf{f}, \mathbf{g}$ Calculation of Lyapunov exponents; $\mathbf{f}$ typical plot of the average log of divergence versus time for the two upper body positions. The lines represent the slopes of the $\log$ (divergence) before a plateau was reached; $\mathbf{g}$ maximum Lyapunov exponent of the dynamic system for the two upper body position. $* p<0.05$ $(n=9 ; t$ test for paired samples)

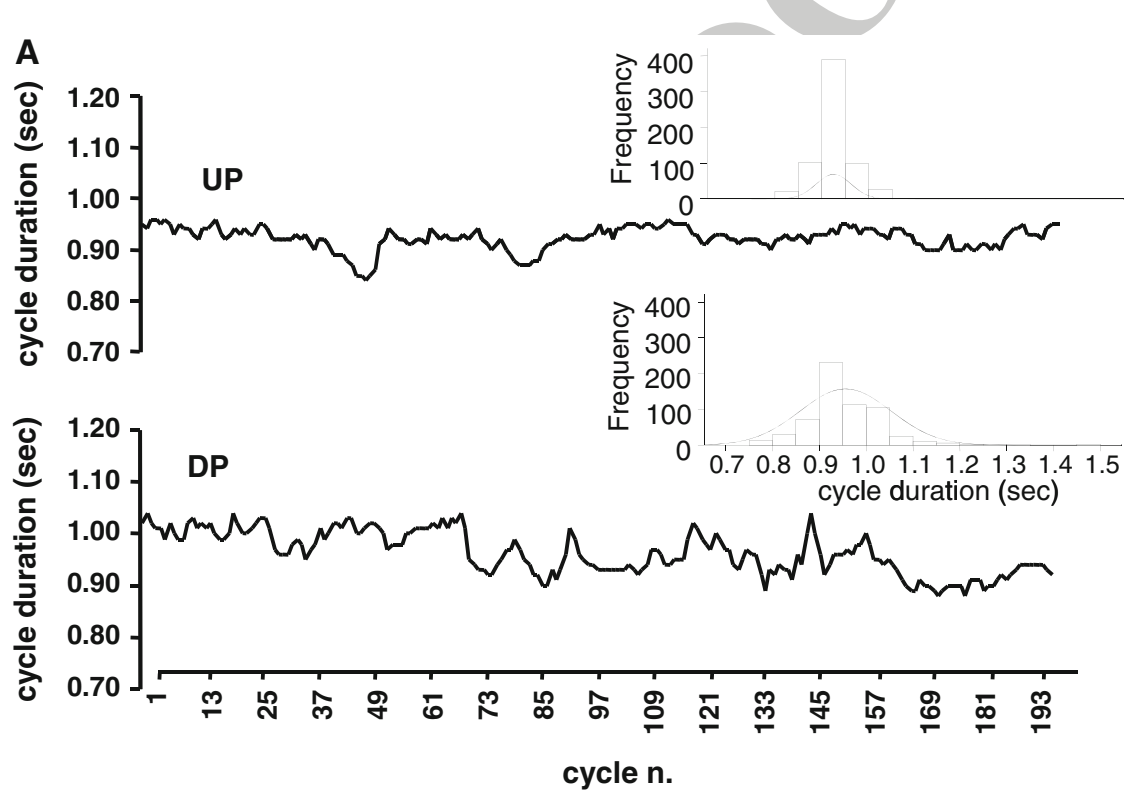

B

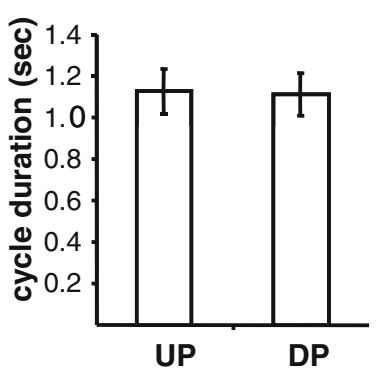

C
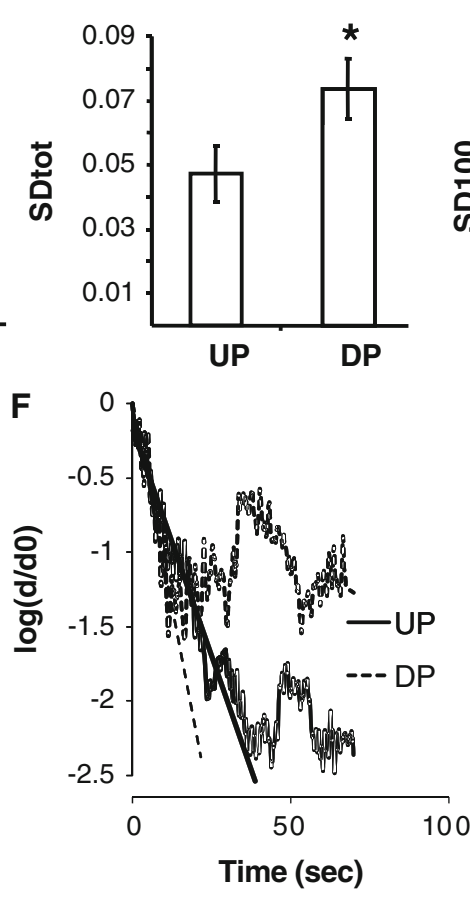

D
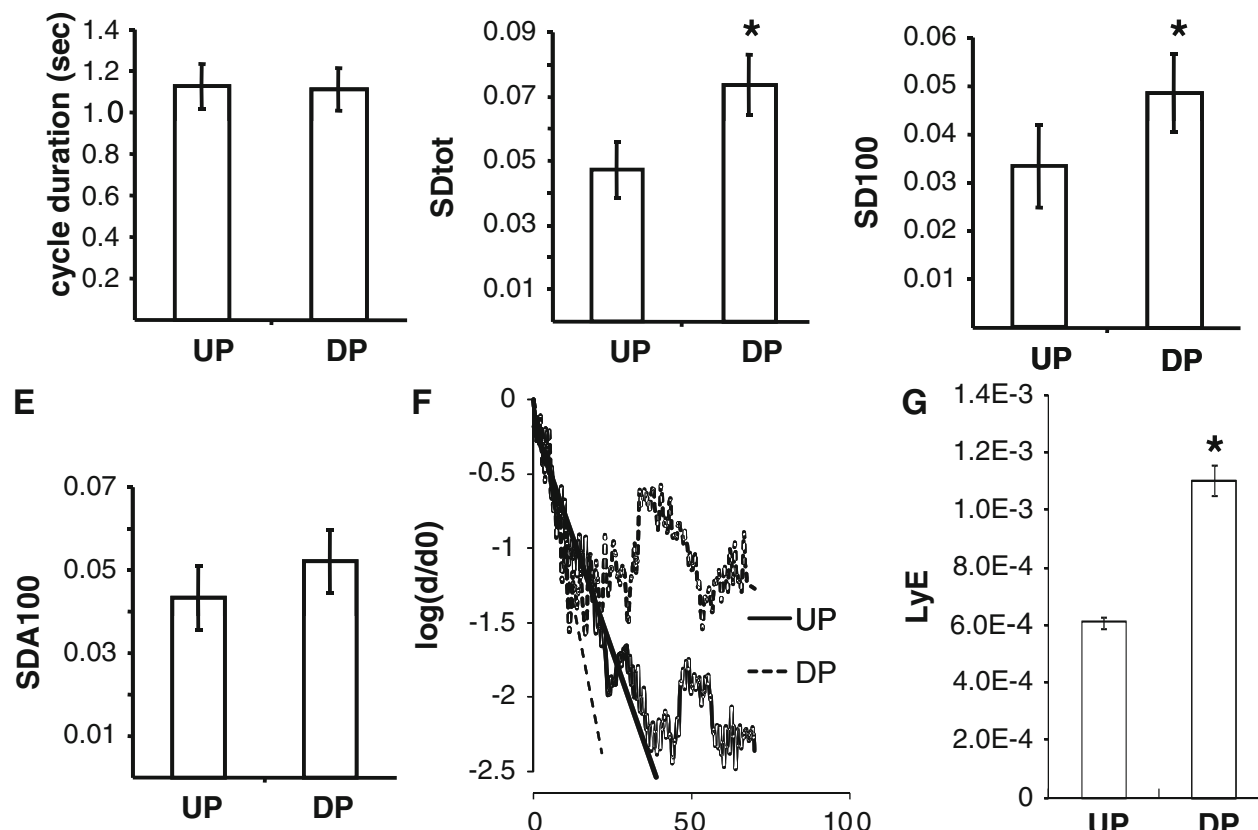

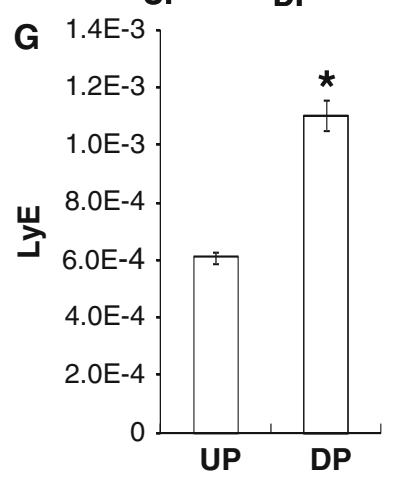


Fig. 2 Heart rate variability during 10-min cycling in two different upper body position (DP hands on lower handlebars, $U P$ upper handlebars).

a Average heart rate during the exercise. b-d Heart rate variability in two upper body positions; b standard deviation of normal to normal $(\mathrm{N}-\mathrm{N})$ intervals (SDNNtot), $\mathbf{c}$ average standard deviation of $\mathrm{N}-\mathrm{N}$ intervals over 100 heart beats (SDNN), d standard deviation of the average $\mathrm{N}-\mathrm{N}$ intervals over 100 heart beats (SDANN) $(n=9)$
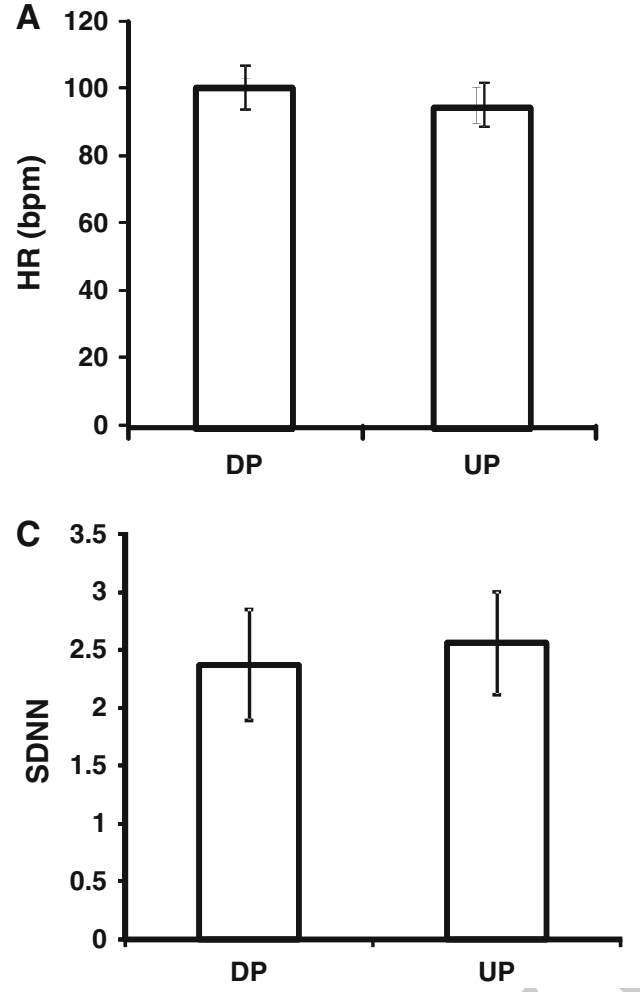
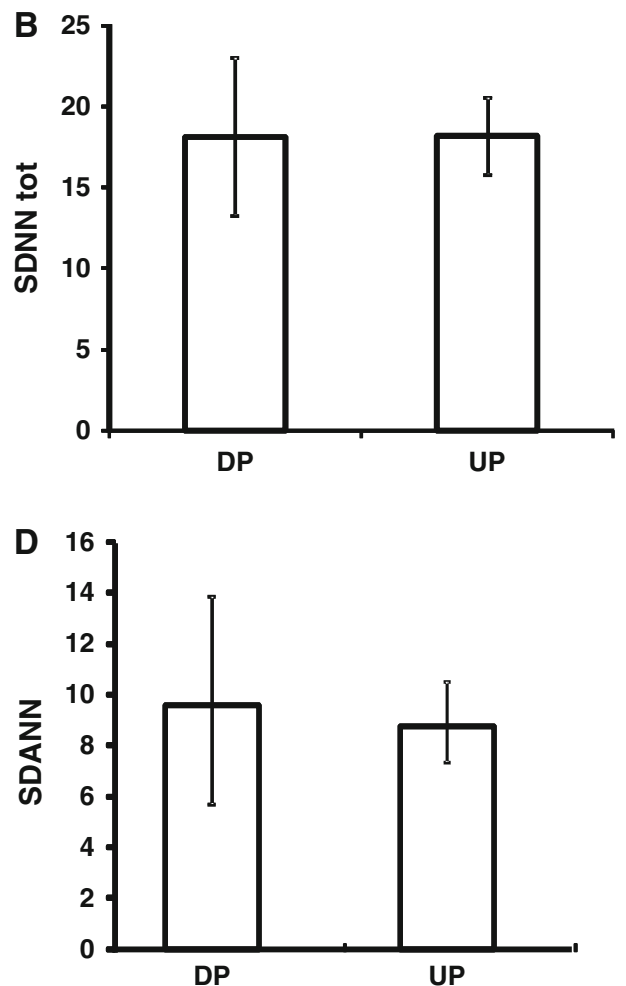

For maximum Lyapunov exponent (LyE) calculation, a 5D embedding space was used after False Nearest Neighbors Statistic. For each data point the minimum distance between orbits $\left(d_{0}\right)$ and the distance after a specific time delay were then calculated $(d)$. The ratio $d / d_{0}$ represented the divergence. In Fig. 1f, a typical plot of the average log of the divergence $\left(d / d_{0}\right)$ versus time for the two upper body positions is represented. To calculate the maximum LyE, the slopes of such $\log$ (divergence) before reaching the plateau have been calculated. Figure $1 \mathrm{~g}$ shows a significant difference of the maximum Lyapunov exponent of the dynamic system for the two upper body positions.

\section{Discussion}

The principal result of the present study is that upper body position influences pedaling time variability during cycling. Previous reports on walking variability demonstrated that several factors such as aging, neuropsychiatric diseases, Parkinson's disease, cruciate ligament deficit (Hausdorff 2009), may influence step duration variability. Therefore, this parameter is of interest to evaluate the integrity of motor systems. However, although pedaling involves cyclic movement of legs there are no data concerning cycling variability. This report demonstrates that the correction of the cycle period can be easily modulated by small changes in the position of upper body, thereby resulting in a greater number of corrections of pedaling time. It was previously shown that, during cycling, the electromyographic (EMG) pattern of lower limb muscles (and particularly of the biceps femoris and tibialis anterior) varies among different individuals and may even change in the same individual during a test (Dorel et al. 2008). This may result in a change of the cycling period.

The analysis of LyE also supports this hypothesis. In fact, in our conditions the LyE is negative, which indicates a deterministic system with an attractor. In other terms, when the system is subject to a perturbation, it tends to return to a stable steady state. In our case, if the rider stops pedaling the resulting evolution of the system converges toward the same state, being dictated by the friction: in general, this is an example of a dissipative system. When comparing the LyE of cycling and walking, the two systems appear quite different: LyE for walking has been estimated to be about 0.14 (Smith et al. 2010), that is a more chaotic regimen, whereas our data show a deterministic system. This strong regularity of cycling behavior is likely due to the fixed circular trajectory of the foot, compared to the inverted pendulum dynamic of walking.

Intriguingly, the dropped posture induces the LyE to become more negative in cycling. It is presently unclear how the change in posture influences pedaling variability, whether this derives from discomfort or from mechanical factors or other physiological/neurophysiological contributions, and carefully designed experiments are needed to

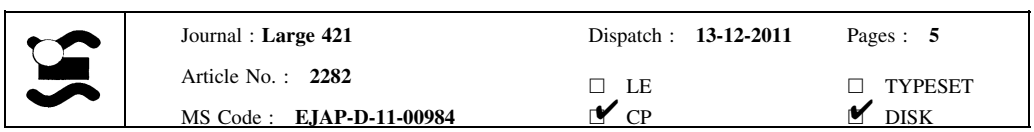


disentangle this question. As suggested by a referee, it seems unlikely that the changes in variability are due to fatigue, because we did not observe changes in heart rate and in heart rate variability. Moreover, the experiments were designed in order to reduce at minimum possible biases in the interpretation of the data deriving from different workloads in the two riding conditions.

The variability of step time is taught to reflect the need of central pattern generators (CPG) to correct timing activation of different muscles across the step cycle. Therefore, it is possible that the increase of the variability in DP is due to an increased number of corrections during the cycle due to the position (Jung et al. 1997; Norris et al. 2011). This is also suggested by the observation that restriction of arm movements changes hip movement variability during walking (Marks 1997).

\section{Conclusion}

Although cycling may be taught as a uniform phenomenon, there is actually some variability in cycle-to-cycle period, probably due to error corrections of cycle timing. We report that cycling variability is increased with a dropped posture, suggesting that in this position a larger number of errors occur. Therefore, cycling variability may be a simple index which could be studied and other physiological and pathological conditions.

Conflict of interest The authors declare that they have no conflict of interest.

\section{References}

Blain G, Meste O, Blain A, Bermon S (2009) Time-frequency analysis of heart rate variability reveals cardiolocomotor coupling during dynamic cycling exercise in humans. Am J Physiol Heart Circ Physiol 296:H1651-H1659

Corbeil P, Blouin JS, Begin F, Nougier V, Teasdale N (2003) Perturbation of the postural control system induced by muscular fatigue. Gait Posture 18:92-100

Dorel S, Couturier A, Hug F (2008) Intra-session repeatability of lower limb muscles activation pattern during pedaling. J Electromyogr Kinesiol 18:857-865

Dorel S, Couturier A, Hug F (2009) Influence of different racing positions on mechanical and electromyographic patterns during pedalling. Scand J Med Sci Sports 19:44-54

Gates DH, Dingwell JB (2008) The effects of neuromuscular fatigue on task performance during repetitive goal-directed movements. Exp Brain Res 187:573-585

Hausdorff JM (2009) Gait dynamics in Parkinson's disease: common and distinct behavior among stride length, gait variability, and fractal-like scaling. Chaos 19:026113

Hausdorff JM, Ladin Z, Wei JY (1995a) Footswitch system for measurement of the temporal parameters of gait. J Biomech 28:347-351

Hausdorff JM, Peng CK, Ladin Z, Wei JY, Goldberger AL (1995b) Is walking a random walk? Evidence for long-range correlations in stride interval of human gait. J Appl Physiol 78:349-358

Jordan K, Challis JH, Newell KM (2007) Walking speed influences on gait cycle variability. Gait Posture 26:128-134

Jung R, Jung J, Losch B (1997) Increased variability in motor output with brain-spinal cord interaction. Biomed Sci Instrum 34:107-112

Marks R (1997) The effect of restricting arm swing during normal locomotion. Biomed Sci Instrum 33:209-215

Meardon SA, Hamill J, Derrick TR (2011) Running injury and stride time variability over a prolonged run. Gait Posture 33:36-40

Norris BJ, Wenning A, Wright TM, Calabrese RL (2011) Constancy and variability in the output of a central pattern generator. J Neurosci 31:4663-4674

Rosenstein MT, Collins JJ, De Luca CJ (1992) A practical method for calculating largest Lyapunov exponents from small data sets. Physica D 65:117-134

Smith BA, Stergiou N, Ulrich BD (2010) Lyapunov exponent and surrogation analysis of patterns of variability: profiles in new walkers with and without Down syndrome. Mot Control 14:126-142

Sterigou N, Decker ML (2011) Human movement science human movement variability, nonlinear dynamics, and pathology: is there a connection? Hum Mov Sci 30:869-888

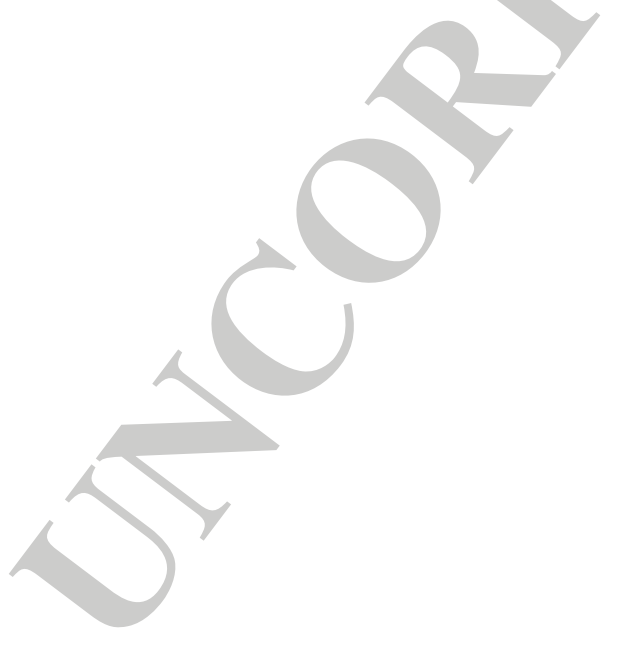

\begin{tabular}{|l|lll|}
\hline & Journal $:$ Large 421 & Dispatch : 13-12-2011 & Pages : $\mathbf{5}$ \\
Article No. : $\mathbf{2 2 8 2}$ & $\square \mathrm{LE}$ & $\square$ TYPESET \\
& MS Code : EJAP-D-11-00984 & $\sim_{\mathrm{CP}}$ & $\checkmark$ DISK \\
\hline
\end{tabular}

